\title{
Optimal navigation and behavioural traits in oceanic migrations
}

\author{
Pinti, Jérôme; Celani, Antonio; Thygesen, Uffe H.; Mariani, Patrizio
}

Published in:

Theoretical Ecology

Link to article, DOI:

$10.1007 / \mathrm{s} 12080-020-00469-4$

Publication date:

2020

Document Version

Peer reviewed version

Link back to DTU Orbit

Citation (APA):

Pinti, J., Celani, A., Thygesen, U. H., \& Mariani, P. (2020). Optimal navigation and behavioural traits in oceanic migrations. Theoretical Ecology, 13, 583-593. https://doi.org/10.1007/s12080-020-00469-4

\section{General rights}

Copyright and moral rights for the publications made accessible in the public portal are retained by the authors and/or other copyright owners and it is a condition of accessing publications that users recognise and abide by the legal requirements associated with these rights.

- Users may download and print one copy of any publication from the public portal for the purpose of private study or research.

- You may not further distribute the material or use it for any profit-making activity or commercial gain

- You may freely distribute the URL identifying the publication in the public portal

If you believe that this document breaches copyright please contact us providing details, and we will remove access to the work immediately and investigate your claim. 


\section{1 optimal navigation and behavioural traits in}

\section{2 oceanic migrations}

3 Jérôme Pinti ${ }^{1, a,{ }^{*}}$, Antonio Celani ${ }^{2, b}$, Uffe H. Thygesen ${ }^{3, c}$, Patrizio Mariani ${ }^{1, d}$

$4 \quad{ }^{1}$ VKR Centre for Ocean Life, National Institute of Aquatic Resources, Technical University of Denmark, 2800 Kongens

5 Lyngby, Denmark

$6{ }^{2}$ The Abdus Salam International Centre for Theoretical Physics, Strada Costiera, 11 I, 34151 Trieste, Italy

$7 \quad{ }^{3}$ DTU Compute, Technical University of Denmark, 2800 Kongens Lyngby, Denmark

8 a Orcid: 0000-0002-0664-0936, ${ }^{\mathrm{b}}$ Orcid: 0000-0002-0339-1073, ${ }^{\mathrm{c}}$ Orcid: 0000-0002-4311-6324, ${ }^{\mathrm{d}}$ Orcid: 0000-0002-

$9 \quad 8015-1583$

$10 *$ Correspondence to jppi@aqua.dtu.dk

11 Key words: Long-distance migrations, migration routes, behavioural traits, decision making, mechanistic

12 modelling, optimal control

14 This manuscript is the preprint of the article published in Theoretical Ecology, accessible here:

15 https://link.springer.com/article/10.1007/s12080-020-00469-4

\section{Abstract}

18 Many organisms perform regular migrations over long distances. These movements are often related to

19 feeding and reproductive periods, and regulated by oceanographic conditions as well as physiological and

20 behavioural traits. Different individual traits and their associated evolutionary constraints will ultimately

21 shape the migratory strategy (and route) of individuals. Optimality theory can provide a framework to

22 assess these inherent trade-offs in individual migrations and identify optimal migration routes in different

23 conditions. Here, we present a model that describes the behavioural trade-off between migration time

24 and energy expenditure and identifies optimal migration routes in realistic ocean conditions. The model

25 explicitly includes a behavioural factor for individual risk management, including risks associated with 
moving in a stochastic environment. We test this model in three different case studies, one in an idealized theoretical context and two in realistic conditions for sea turtle migrations. We show that behavioural traits can largely influence the optimal routes in long-distance migrations, resulting in major changes in migratory pathways. Further, we assess the ability of the model to infer back behavioural traits given a set of synthetic individual tracks and show relatively good performances. However, further tests are needed to evaluate performances when accurate observations of migrations are used.

\section{Introduction}

Migrations are common phenomena in terrestrial and aquatic ecosystems typically associated to the most important tasks in the organisms' life history: feeding, survival and reproduction (Dingle 2014). In some groups of birds, marine mammals and fish, these migrations can span thousands of kilometres literally covering the entire planet (Alerstam et al. 2003; Block et al. 2011). Despite the strong selective pressures on migrations, diverse strategies can coexist even within population of the same species including partial migration, different time of departures, distinctive destinations, number of stopovers, moving speed, etc. (Alerstam 2001; Byron and Burke 2014). The existence of these widely variable migration strategies has generated questions regarding the adaptive value of migrations and the relative importance of ecological and evolutionary processes in shaping them (Alerstam et al. 2003; Cardona et al. 2017; Dalleau et al. 2019). The details of migration patterns have been studied for a range of marine species (see e.g. Nichols et al. 2000; Bonfil et al. 2005; Aarestrup et al. 2009; Block et al. 2011), and several mechanisms have been suggested for both proximate and ultimate causes driving such processes. Genetic, physiological and behavioural adaptations are considered key attributes for the evolution of migrations (Alerstam et al. 2003; Dingle 2014; Lennox et al. 2016). Generally, migrants undergo these long journeys to find more favourable conditions such as better food, reduced predator pressure, better environmental conditions (Alerstam et al. 2003; Shaffer et al. 2006; Block et al. 2011). The knowledge of migration routes can be innate, learnt through natal homing or taught by other individuals. (Fagan et al. 2013; Mueller et al. 2013; Scott et al. 2014). Specific physiological adaptations have been identified in long distance migrants which can expose them to potential vulnerabilities under environmental disturbances including climate change (Feder et al. 2010; Lennox et al. 2016). On the other hand, plasticity in behavioural traits can directly affect migration routes adapting them to changing food availability, temperature and ocean currents (Jørgensen et al. 2006;

54 Block et al. 2011; Hays et al. 2014b) . 
55 In particular, advection by ocean currents can have a large impact on the realized migration routes 56 (Thomson et al. 1992; Luschi et al. 2003; Metcalfe et al. 2006; Hays et al. 2014a). The impact can be either 57 negative in case of counter-current or lateral drift, or positive when favourable ocean currents allow 58 reaching the destination faster and/or with less energy. While it is well known that migrating birds can 59 adjust their speed in response to local wind conditions (i.e., increasing it in headwinds and reduce it in 60 tailwinds (Bloch and Bruderer 1982)), mechanisms for navigation and active control of speed and heading 61 in marine organisms are less clear (Hays et al. 2003; Åkesson and Hedenström 2007).

62 Optimality methods can be used for the analyses of behavioural strategies and adaptations in the long63 distance migrations of several species (Hedenström 2003; Alerstam 2011) but presently few applications 64 can be found for marine organisms (but see Hays et al. 2014a). An optimality approach may assist in 65 providing reference solutions to compare against observed migrations tracks and to infer whether specific 66 individual behaviour is directed towards saving energy, reduce migration time, avoid competition and 67 predators, maximize reproduction, etc. (see e.g. Carmel and Ben-Haim (2005) in the case of foraging 68 theory). In the case of movements in ocean currents the optimality solution to minimize migration time is analogous to solving Zermelo's navigation problem (Zermelo 1931) where a boat crossing a shear flow at constant speed can reduce navigation time by deviating its route from a simple straight line linking initial and final destination points. However, animal migrations are subject to additional physiological and behavioural constraints that could modify the identification of optimal tracks when only a minimization of migration time is considered.

In this paper, we present a minimal theoretical mechanistic framework to investigate optimal migratory routes in relation to ocean currents, behavioural and physiological constraints. The model links a description of different behavioural traits to the external factors during migration (e.g. ocean currents and turbulence) and provide the optimal navigational strategy in a stochastic environment. This approach is a generalization of Zermelo's navigation problem where noise as well as animals' behavioural and physiological constraints are specifically included.

80 The model is illustrated for the case of sea turtle migrations in realistic conditions and contrasting ocean 81 regions, where detailed description of migration routes are available in the literature. Hundreds of random 82 tracks are generated in those areas, and the model outputs are compared with available observations.

83 Further, the model is used to infer back the underlying behavioural traits shaping such migration routes, 84 demonstrating skills and limitations of the proposed approach. We suggest that the optimality model 
presented here could be combined with high-resolution animal tracking technologies to gain insight about the specific behaviour ecology of the migrants.

\section{Methods}

\subsection{Model formulation}

92 We consider a minimal model for the migration of single individuals that are able to swim and adjust their 93 velocity based on local ocean current conditions. We model the local ocean currents by the sum of a mean 94 velocity component (i.e., $V(X)$ ) and a white-noise process with diffusion coefficient $D$. The latter represents 95 small scale fluctuations associated to e.g., mesoscale and/or ocean turbulence processes. We further assume that an organism can control its movement and swim at the control velocity $w$. Then, the dynamics

97 of the migrant can be described by the following Itō stochastic differential equation ( $\varnothing$ ksendal 2003):

$$
d X_{t}=\left(v\left(X_{t}\right)+w\left(X_{t}\right)\right) d t+\sqrt{2 D} d B_{t}
$$

where $X_{t}$ is the position of the organism in two dimensions at time $t$, while $B_{t}$ is two-dimensional standard Brownian motion. For long-distance regular migrants, evolutionary constraints will benefit behavioural and physiological traits able to minimize the time needed to reach the target while limiting the energy spent on migration. Hence, we can define the total cost of travelling to the target:

$$
C=\frac{\gamma}{2} \int_{0}^{T}|w(X(t))|^{2} d t+\beta T,
$$

where $T$ is the total duration of the migration event and $\beta$ and $\gamma$ are the parameters defining the trade-off between time and energy, respectively. This cost explicitly describes the trade-off between time taken to reach the target and the energy expenditure required to swim at the control velocity $w$. We assume that the energy expenditure related to swimming scales with the square of the control speed which seems to be consistent with experimental data from turtles (Prange 1976). Other metabolic expenses (e.g., basal metabolism) are assumed to be a linear function of time. We do not consider energy acquisition during the migration (or only implicitly and at a constant rate). Within this framework, when the flow is absent, 
the optimal control is trivially a straight line from the starting point to the target, but when the flow field

110 is present the optimal control can lead to a variety of trajectories (Bryson and Ho 1975; Comincioli 2010;

111 Hays et al. 2014a). Zermelo's problem was formulated as a deterministic control, while we consider here

112 a stochastic component to account for both the variability of the ocean currents (e.g. mesoscale dynamics)

113 and for the noise arising from imperfect decisions of the migrants.

114 The cost $C$ in Eq. 2 is random so the navigation strategy should minimize some statistic of the cost. Here,

115 we introduce a risk-parameter $\alpha$ that accounts for the specific risk-sensitivity of the organism. Indeed, 116 different individuals could weight differently the risk associated in moving under uncertainty conditions 117 and a combination of risk-averse or risk-prone strategies could emerge (Wolf et al. 2007). This is similar to 118 economic theory when agents need to consider the long-term implications of their instant decisions and 119 could employ strategies to minimize the risks of worst case scenarios (Whittle 1990). To introduce a risk120 sensitivity trait in the model we hypothesize that the organism aims at minimizing (Howard and Matheson 121 1972):

$$
F=\frac{1}{\alpha} \log \langle E[\exp (\alpha C)]\rangle
$$

122 This risk-sensitivity term adds an additional parameter changing the weight of the best or worst possible 123 occurrences. As $\alpha$ tends to zero, we recover the risk-neutral strategy where the expected cost $E[C]$ is 124 minimized. However, if $\alpha$ is small but finite, a Taylor's expansion shows that the function $F$ becomes a 125 linear combination of mean cost and variance, with $\alpha$ weighing the importance of the latter with respect 126 to the former. In general, $\alpha>0$ corresponds to a risk-averse strategy, where outcomes with high values of 127 the cost are penalized heavily so that the optimal strategy focuses on these worst-case scenarios (the goal 128 is to decrease the variance). Conversely, $\alpha<0$ corresponds to a risk-seeking attitude where the few best129 case scenarios are rewarded (the variance is valued).

130 Given the three behavioural traits $\alpha, \beta$ and $\gamma$ the cost $F$ can be computed at all points of the ocean basin 131 using Dynamic Programming (Bellman 1954; Øksendal 2003). $F$ is then given as the solution to the 132 following Hamilton-Jacobi-Bellman equation:

$$
v \cdot \nabla F+\left(D \alpha-\frac{1}{2 \gamma}\right)|\nabla F|^{2}+D \nabla^{2} F=-\beta,
$$

133 with boundary conditions $\mathrm{F}=0$ at the target and reflective (Neumann) boundary conditions elsewhere.

134 This equation can be rewritten using the transformation (Dvijotham and Todorov 2011): 


$$
F=\frac{1}{\delta} \log (Z), \text { where } \delta=\alpha-\frac{1}{2 D^{\prime}}
$$

135 then leading to the following partial differential equation (PDE):

$$
v \cdot \nabla Z+D \nabla^{2} Z+\delta \beta Z=0
$$

136 Once this equation is solved, the optimal control velocity at any point can be found using the gradient of

137 the cost:

$$
w=-\frac{1}{\gamma} \nabla F
$$

138 The derivation of these equations is justified in more details in supplementary material S1. Eq. 6 is solved using COMSOL (COMSOL Multiphysics ${ }^{\circledR}$ v. 5.2. www.comsol.com. COMSOL AB, Stockholm, Sweden) 140 coupled to MATLAB 2016b (MATLAB 2016b, The MathWorks Inc., Natick, MA, 2000, United States of 141 America) via Livelink. The code needed to run the model described here is available as supplementary 142 material.

\subsection{Reconstruction of behavioural parameters}

144 The objective of the statistical inference is to estimate the behavioural parameters based on observed 145 tracks of the organisms, i.e. sets of points $\xi=\left(X_{i}, t_{i}\right)_{i \in[1, N]}$, where $X_{i}$ is the position of the organism at the time $146 \mathrm{t}_{\mathrm{i}}$. For a given set of behavioural traits, the following quasi log-likelihood is defined:

$$
L(\xi \vee \alpha, \beta, \gamma)=-\sum_{i=1}^{N-1}\left\|\left(X_{i+1}-X_{i}\right)-\left(v\left(X_{i}\right)+w\left(X_{i}\right)\right) \cdot\left(t_{i+1}-t_{i}\right)\right\|^{2}
$$

This log-likelihood function measures the difference between the measured speed vector and the theoretical speed vector at each point of the track. The log-likelihood is maximized numerically with a Nelder-Mead algorithm (Lagarias et al. 1998), thus identifying behavioural traits that fit the migration event the best.

Note that the two parameters we aim to reconstruct are $\alpha$ and $\beta / \gamma$. This is because varying $\beta$ and $\gamma$

152 together is redundant when $\alpha=0$ and only the cost ratio $\beta / \gamma$ matters. Conveniently, this ratio is also the 153 most interesting value in an ecological context representing the relative allocation between swimming and metabolic expenditures. Similarly, we are interested in identifying the main patterns in risk-averse or riskseeking behaviour, hence we only focus on the sign of $\alpha$ more than on its absolute value. Indeed the exact value of $\alpha$ is difficult to associate to specific life-history characteristics and can also be expected to rapidly change over time. 
When $\alpha$ is very small, we recover the cost ratio $C$ from eq. (2) and thus the control $w$ is invariant if we multiply both $\beta$ and $\gamma$ by a constant $\lambda$ :

$$
w=\frac{-1}{\lambda \gamma} \nabla(\lambda F)=\frac{-1}{\gamma} \nabla F .
$$

The diffusivity $D$ is given and considered constant throughout the migration event. A value of $D=$ $1621000 \mathrm{~m}^{2} \cdot \mathrm{s}^{-1}$ is used corresponding to an average horizontal turbulent diffusivity in the oceans (Cole et 163 al. 2015). The effects of different values of the diffusion term have been analysed in more details in the 164 supplementary material. Eq. 8 has been selected after testing different log-likelihood functions including 165 those reconstructing the diffusivity values. We selected the log-likelihood function that reconstructed the behavioural traits in the theoretical case with the smallest error.

To test the method, artificial trajectories with random behavioural traits were generated. Then behavioural traits could be inferred using eq. (8) and compared with the initial ones to evaluate the accuracy and precision of the reconstructing method. $\alpha$ could vary between -10 and 1 , and $\beta / \gamma$ between $10^{-2}$ and $10^{2}$.

171 To better compare the reconstruction between different scenarios, the cost ratio has been 172 adimensionalised, meaning that it is defined as $\beta /\left(\nu|w|^{2}\right)$, with $|w|$ the mean control speed during the 173 migration. For each reconstructed parameter $X$ ( $\alpha$ or the adimensional cost ratio), we calculate the 174 reconstruction error as $\frac{X_{\text {generated }}-X_{\text {reconstructed }}}{X_{\text {generated }}}$.

\subsection{Case studies}

Three case studies have been investigated ranging from purely theoretical to more realistic scenarios: (1)

178 organisms crossing a shear flow $\left(\vec{v}=c \overrightarrow{e_{x}}\right)$ from different starting points; (2) migrations of sea turtles from 179 Ascension Island to the Brazilian coast mimicking observations on green turtles (Luschi et al. 1998); (3) sea 180 turtle migrations from Diego García in the Indian Ocean to the Somalian coast (a migration route that was 181 also observed for green turtles (Hays et al. 2014b)). The last two study cases used realistic oceanic 182 conditions, with averaged ocean currents corresponding to the relevant migration periods obtained from 183 the Operational Mercator global ocean analysis and forecast system (available at 184 http://marine.copernicus.eu) with a frequency of one day and a spatial resolution of $10 \mathrm{~km}$. The oceanographic current field considered is the multi-year average (2007-2016) surface currents in the 
region during the migration windows of the green turtles. To this average current velocity, we added the diffusivity term modelled as in Eq. 1. For the the Atlantic Ocean case, the migration window considered was between April and August (Luschi et al. 1998), and for the study case in the Indian Ocean between October and February (Hays et al. 2014b).

\section{Results}

\subsection{Track generation}

\subsubsection{Crossing a simple shear flow}

Different behavioural traits values $(\alpha, \beta, \gamma)$ generate substantially different trajectories when crossing a shear flow (Figure 1). Typically, lower values of $\alpha$ yield longer migration routes, as individuals would reduce their instantaneous cost to a minimum and swim actively only when it is necessary to reach the final destination. Risk-averse individuals tend to avoid regions of high currents pointing towards unfavourable directions. There is an inverse proportionality between $\beta$ and $\gamma$ hence their ratio regulate the shape of the migration route. A high ratio $\beta / \gamma$ means that the optimal strategy is achieved by reducing the migration time more than minimizing energy expenditures. Thus with high $\beta / \gamma$ organisms should rely less on the currents, resulting in trajectories that are generally straighter and with a higher control speed, hence shorter navigation time. Varying $\gamma$ with a fixed $\beta$ would give the same results as varying $\beta$ with a fixed $\gamma$ (Figure 1).

\subsubsection{Migration from Ascension Island to Brazil}

Using random values for $(\alpha, \beta, \gamma)$ in an Atlantic Ocean setup and starting point on Ascension Island, we obtain tracks converging nicely to the Brazilian coast which is the assumed target destination region. The geographical locations of these tracks vary from northern routes (using the fast equatorial current in the north) to southern migrations with a course correction as approaching the Brazilian region. The simulated tracks resemble those observed in the feeding migration of green turtles although model tracks are generally straighter than the ones recorded. Indeed, some turtles have been observed to arrive at more northern regions than our tracks, making use of the southern edge of the Equatorial current (Luschi et al. 1998). The obtained mean control speed in our simulations is in the range $0.74-1.69 \mathrm{~km} / \mathrm{h}\left(1^{\text {st }}\right.$ and $3^{\text {rd }}$ quartile, median $1.58 \mathrm{~km} / \mathrm{h}$ ) with the fastest migration at $3 \mathrm{~km} / \mathrm{h}$ on average (supplementary material). 
214 Interestingly, these optimal migration speeds are consistent with recorded transit speeds for green turtles 215 from Ascension Island (0.9 and $3.1 \mathrm{~km} / \mathrm{h}$ from Luschi et al. (1998) and Akesson et al. (2003)).

216

217

218

\subsubsection{Migration from Diego García to Somalia}

219 The monsoon-driven circulation in the Indian ocean (Schott and McCreary 2001) yields average ocean 220 currents having a more complex spatial structure than those in the Atlantic Ocean case study. As a result, 221 small changes in the behavioural traits can result in large displacement of the optimal migration tracks (Fig. 222 3). Migrations are generally northwards but with different latitudinal gradients, resulting in routes close 223 to a straight line to those displaying several course corrections patterns. The recorded migration patterns 224 of green turtles in the Indian Ocean vary a lot indicating the potential role as milestones of the many small 225 island in the region (Hays et al. 2014b). This factor-together with course correction driven by other 226 environmental cues (e.g., temperature and salinity gradients, geomagnetic field (Akesson et al. 2003; 227 Åkesson and Hedenström 2007)) - is not included in the model, but on a qualitative level the simulated 228 tracks are consistent with the observations. Optimal control speeds are somewhat low in this region $\left(1^{\text {st }}\right.$ 229 and $3^{\text {rd }}$ quartiles are 0.62 and $0.92 \mathrm{~km} / \mathrm{h}$ ), which is slower than the total speeds recorded in the area for 230 migrating turtles, between 2 and $2.8 \mathrm{~km} / \mathrm{h}$ (Hays et al. 2014b). 


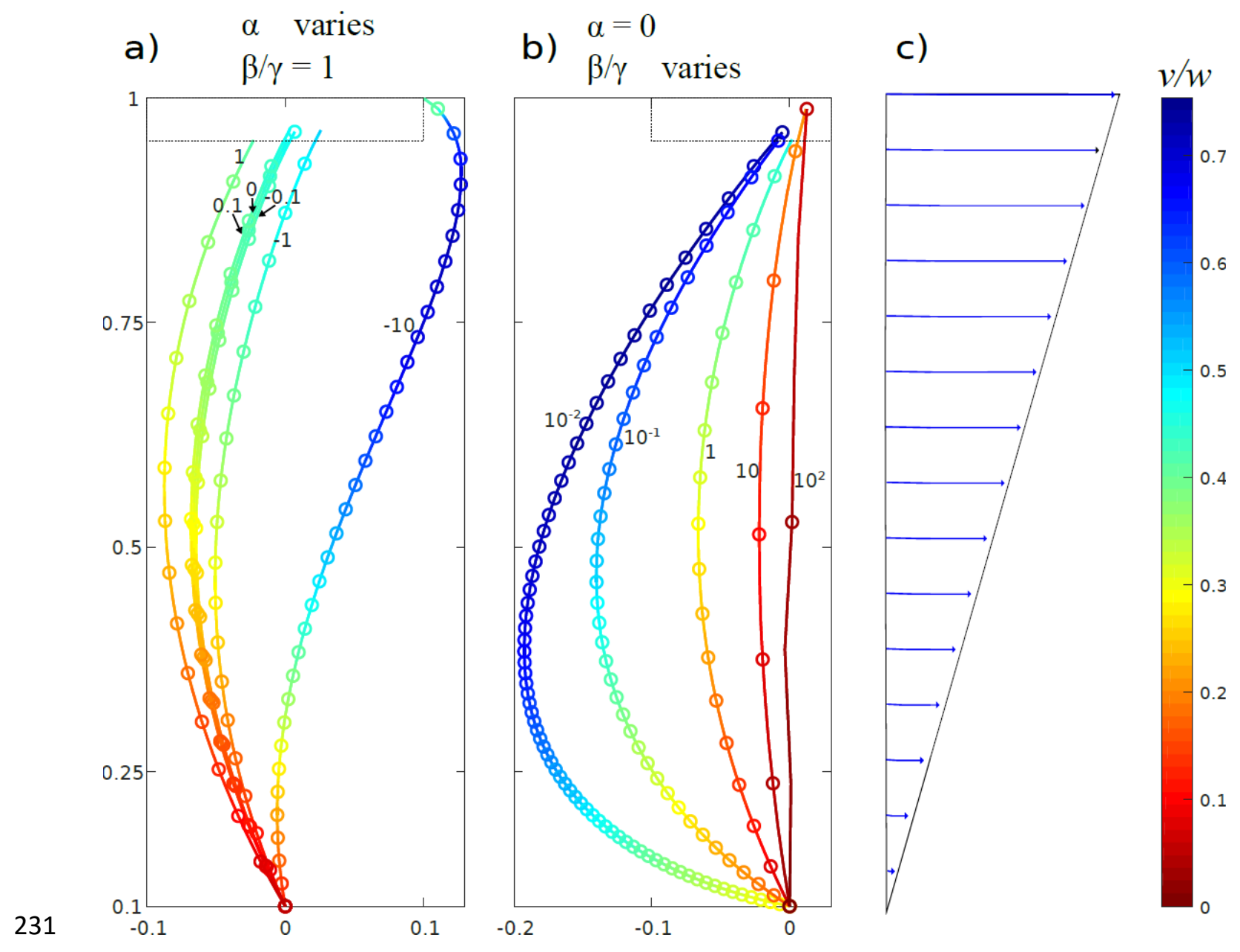

Figure 1 Tracks of migrants crossing a $1 \mathrm{~km}$-long shear flow. The time between two data points is 1 minute, and the colour code shows the ratio between advective and control speed $v / w$. a) Tracks for different values of $\alpha: 1,0.1,0,-0.1,1,10$ (from left to right). b) Tracks for different values of $\beta / \gamma: 10^{-2}, 10^{-1}, 1,10$, 100 (from left to right). c) Representation of the shear flow used. 


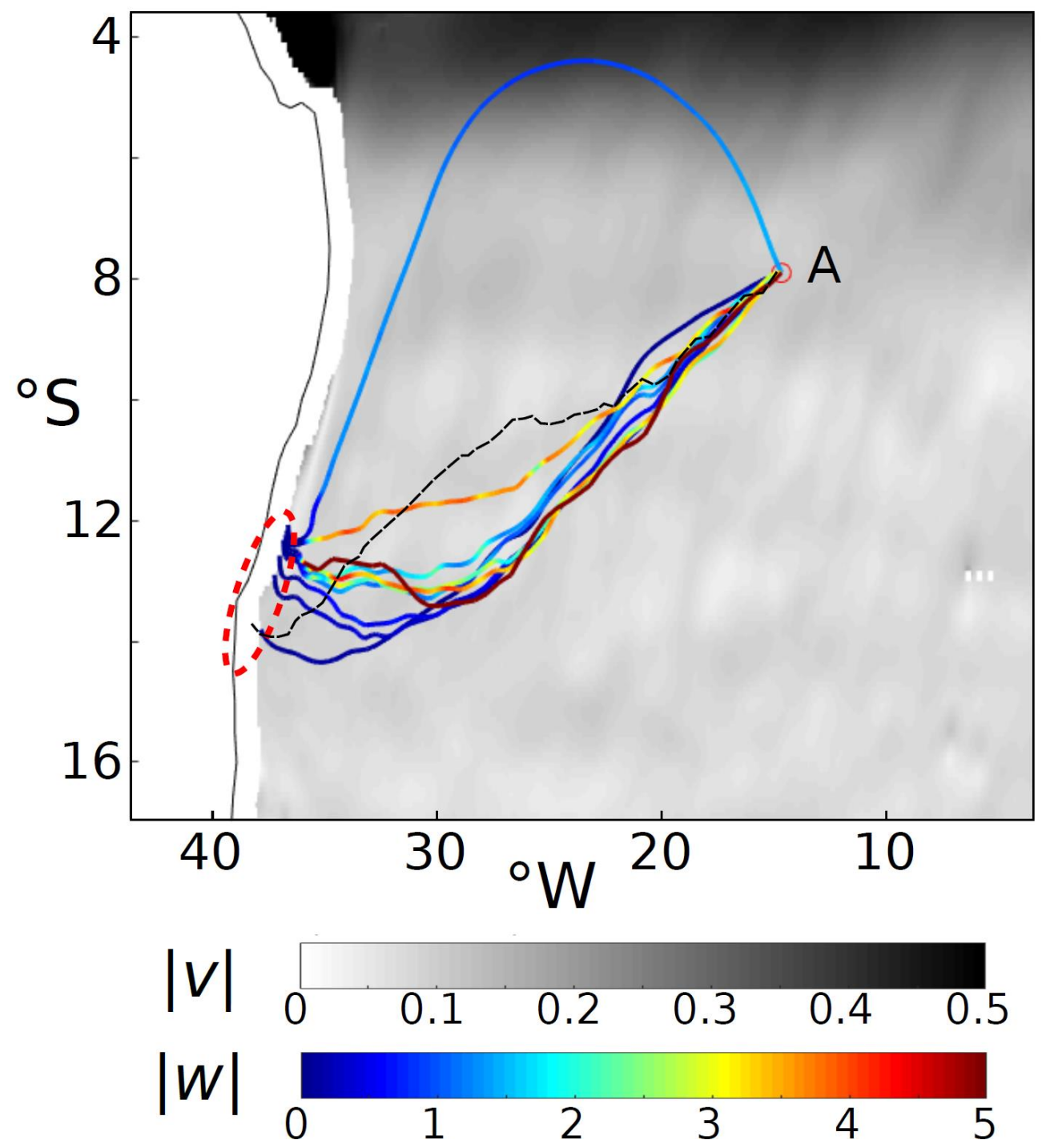

Figure 2 Tracks generated by the model in the Atlantic with random behavioural parameters. The arrival area is the red ellipse, and the departure points is the red circle (A, Ascension Island). Background is the current speed (in $\mathrm{m} / \mathrm{s}$ ), and the track colours represent the control velocity (in $\mathrm{m} / \mathrm{s})$. Dashed black line is an original migration track of green turtle, reproduced from Cerritelli et al. (2019). 


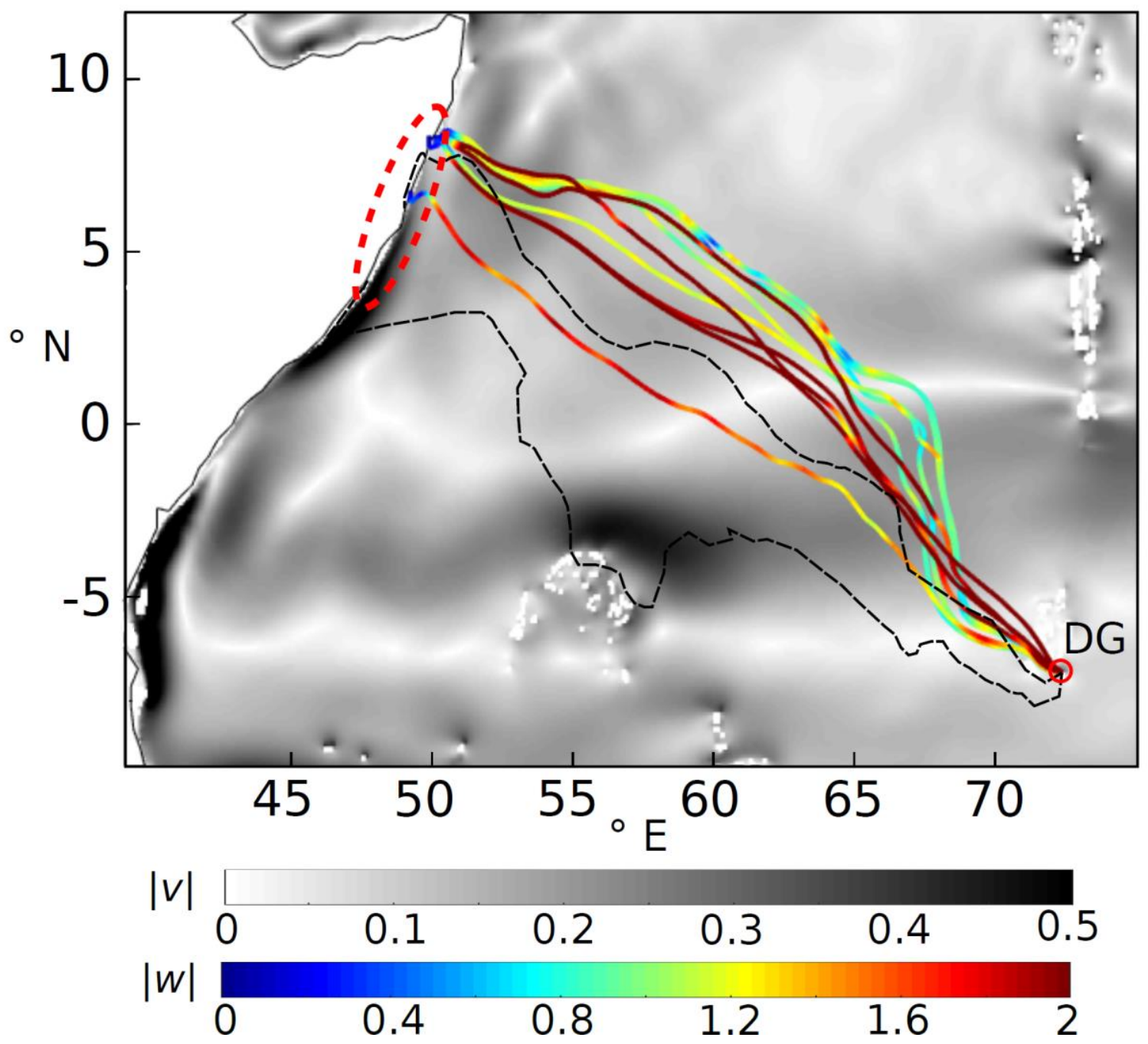

Figure 3 Tracks generated by the model for organisms migrating between Diego García (DG, red circle C) and the Somalian coast (red dashed ellipse) with random behavioural parameters. Background is the current speed (in $\mathrm{m} / \mathrm{s}$ ), and the track colours represent the control velocity (in $\mathrm{m} / \mathrm{s})$. Dashed black lines are original migration tracks of green turtles, reproduced from Hays et al. (2014b).

\subsubsection{Reconstruction for the theoretical case}

Out of the 100 Monte-Carlo simulations of the model with different behavioural traits, some do not lead to viable trajectories. Moreover, a few iterations of the reconstruction algorithm do not converge. In total, 
out of 100 simulations, 90 to 93 converge to a proper migration track and lead to a successful reconstruction.
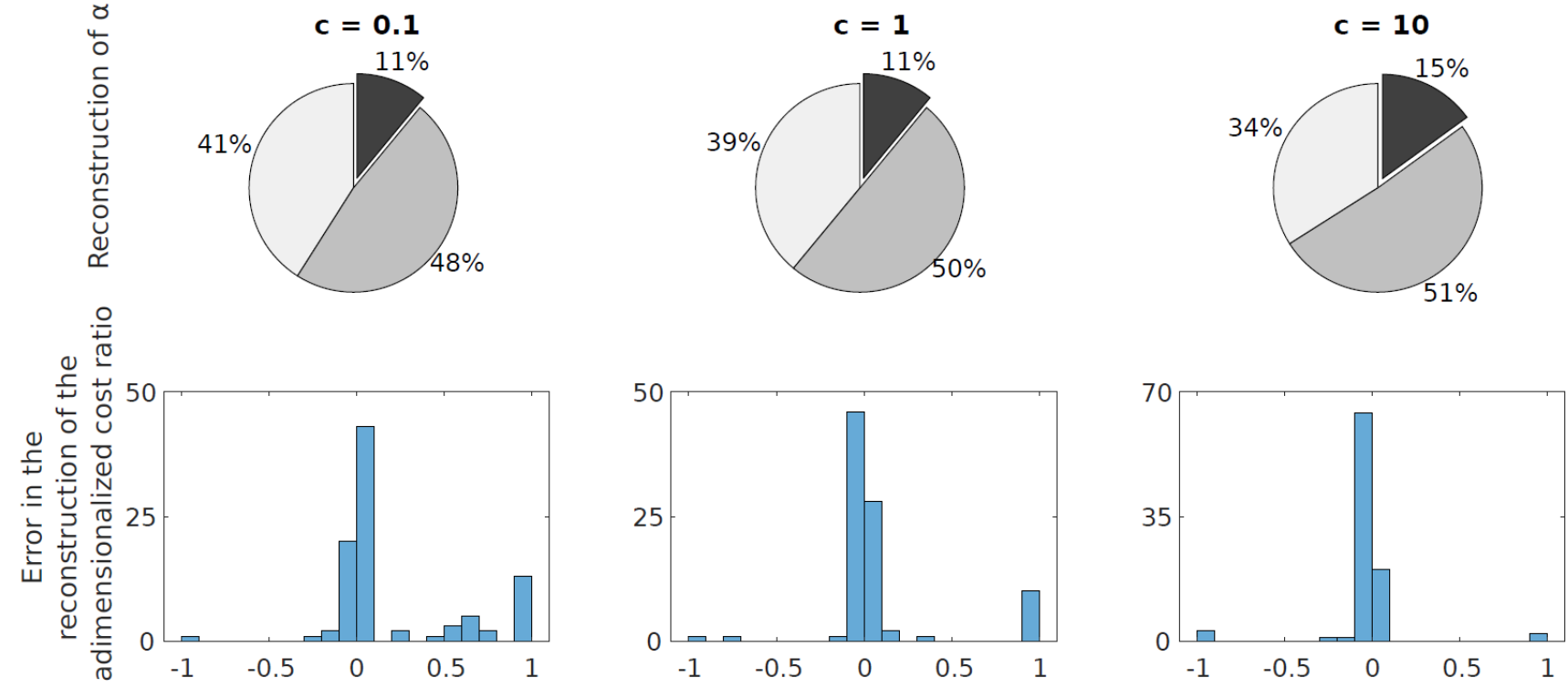

Figure 4 Top: Reconstruction of the sign of the risk parameter $\alpha$ and of the adimensionalized cost ratio in the theoretical cases. For the risk attitude: the dark share of the chart represents the misreconstructed signs, the dark grey the correct positive (risk-averse) reconstruction, and the light grey the correct negative (risk-seeking) reconstructions. Bottom: For the cost ratio: the histograms were constrained between -1 and 1 , and all more extreme values were assigned to -1 (if negative) or 1 (if positive). Each bar represents $10 \%$ of error.

\subsubsection{Reconstruction for oceanic migrations}

The reconstruction of the risk-attitude is better than in the theoretical case, with between 94 and $98 \%$ of correct reconstructions. The accuracy of the inference of the cost ratio reconstruction is also high: $72 \%$ of the tracks in the Atlantic Ocean (80\% in the Atlantic Ocean) lead to reconstructions within $10 \%$ accuracy.

251 However, despite these relatively good performances for such cases, there are a few outliers. For example, in the Atlantic case, $18 \%$ of the reconstructions lead to a cost ratio with an error of more than $200 \%$. 

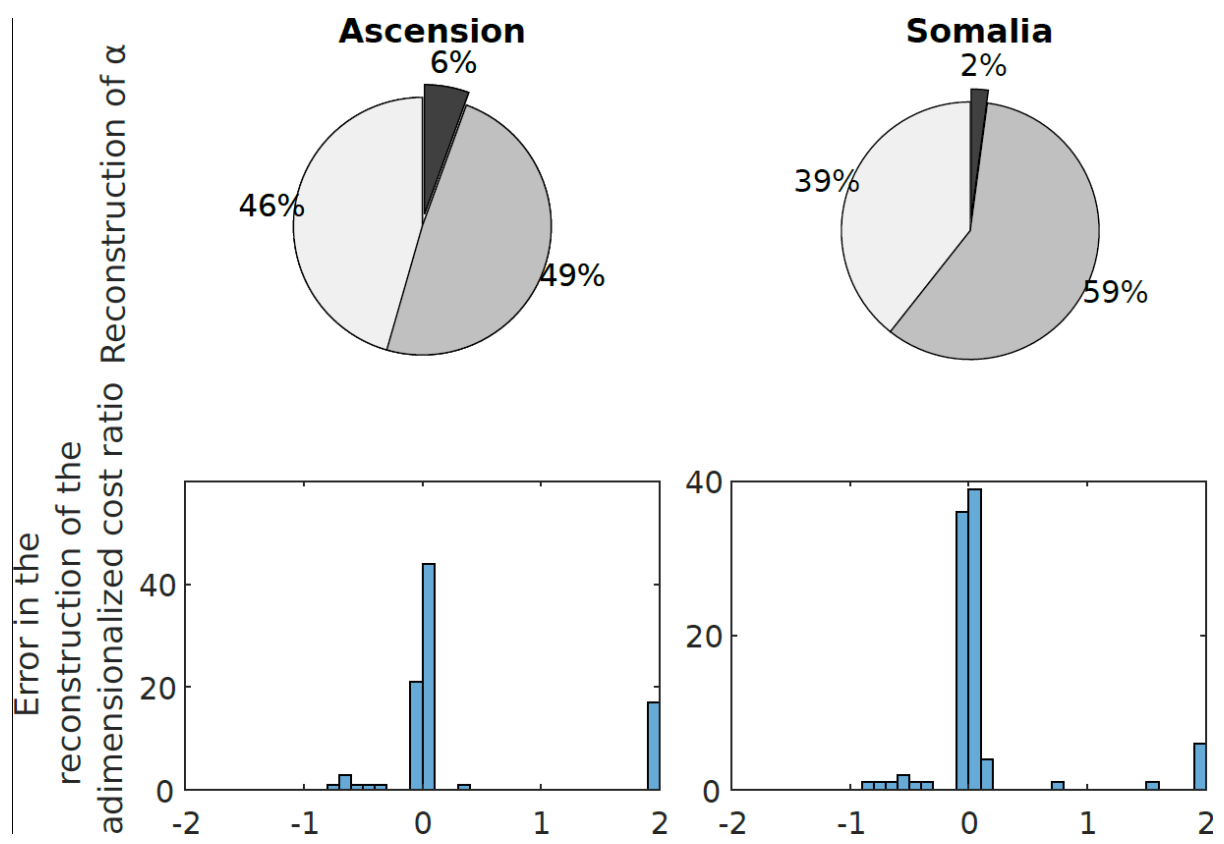

Figure 5 Top: Reconstruction of the sign of the risk parameter $\alpha$ and of the adimensionalized cost ratio in the oceanic cases. For the risk attitude: the dark share of the chart represents the misreconstructed signs, the dark grey the correct positive (risk-averse) reconstruction, and the light grey the correct negative (risk-seeking) reconstructions. Bottom: For the cost ratio: the histograms were constrained between -2 and 2, and all more extreme values were assigned to -2 (if negative) or 2 (if positive). Each bar represents 10\% of error.

\section{Discussion}

256 We introduced a simple model to simulate optimal migrations under different behavioural traits and environmental conditions. The model makes explicit the trade-off between energy saving strategies and arrival time and includes the effects of navigational errors and ocean mesoscale, all considered important aspects in animal movements (Luschi et al. 2003; Åkesson and Hedenström 2007; Shamoun-Baranes et al. 2010). Additionally, a risk-averse behavioural trait is introduced to identify strategies minimizing the risk of incurring in large (i.e., above average) costs during the migrations. A few other works have explored Zermelo's problem in relation to migration before (e.g., in turtles (Hays et al. 2014a) or birds (McLaren et al. 2014)), but they did not consider time and energy constraints together and the different optimal strategies that may arise due to behavioural discrepancies between individuals. Additionally we note that we do not impose the swimming speed, but both navigation speed and headings are output of the model. 
Our model generates optimal migration strategies under different behavioural and oceanographic scenarios. When applied to simulate sea turtle migrations in realistic conditions, the model is able to reproduce some of the patterns observed by GPS-tracking. But not all behavioural strategies can lead to viable migrations, as animals cannot reach the target or the target is reached but outside a suitable time window. When provided with a large set of simulated tracks, the model can also be used to infer back the behavioural traits of the organism. Inference of these behavioural traits is proven to be often accurate but errors in the reconstruction are also reported. The model can provide new insight in the behavioural ecology of long distance migrations when used in combination with accurate high-resolution data on animal tracks.

\section{Learning and optimization of migration routes}

The way organisms select their migration route varies largely between species making it difficult to identify common mechanisms (Mueller et al. 2013; Dingle 2014). However, in different groups, individual learning, social learning and genetic constraints are all considered important components of successful migrations. For example, humpback calves learn their routes during their first years, while they are migrating with their mothers (Clapham and Mayo 1987). This results in later-stage migrations towards mother-defined feeding grounds (Weinrich 1998). Moreover, since whales migrate in pods (at least the females) they can possibly benefit from the social transmission of knowledge (Weinrich 1998), as it has been also hypothesized for other species such as tuna (De Luca et al. 2014) and birds (Mueller et al. 2013). Opposite to the mechanism of learning via social information transfer, turtle hatchlings are completely independent during their first migration (Carr 1987). Hence most likely they have limited, if any, social information about the migration route to take and the hypothesis is that they might rely on the initial dispersal phase to learn migration pathways between nesting site and feeding areas (Hays et al. 2014b; Scott et al. 2014).

Regardless of how marine migrants learn their destination, it seems reasonable to assume that strategies minimizing migration time and energy expenditure are favoured in repeated migration events, as it has been found in birds and ungulates for example (Alerstam and Lindström 1990; Alerstam 2001; Bischof et al. 2012). However, results for adult turtles demonstrate that they are not following routes minimizing migration time alone (Hays et al. 2014a). Still they follow routes that are not far from this optimum and able to bring them to their goal. This could indicate that turtles have evolved a successful migration strategy to cope with the year-to-year variability of the ocean circulation, making it interesting to explore 
optimal migration strategy against multi-year average ocean conditions. It has been suggested that migrating adult turtles cannot effectively perceive current deflection or that their navigation map is not accurate enough to allow optimum route finding (Hays 2017). Here we suggest that including swimming cost and/or differences in individual behaviour could provide a more general framework to assess optimality conditions in long-distance migrants.

Ocean currents are highly variable in space and time hence optimization should work on expected conditions over repeated migrations. In the model presented here, we considered average ocean current conditions in the relevant migration period and include a random term to simulate mesoscale activity or likewise to describe year-to-year variability. This should be conditions relevant for the emergence of optimal migration strategies in an environment with known stochasticity.

The relationship between expected value and variability is introduced as an additional parameter in the model ( $\alpha$, Eq. 3). If $\alpha$ is zero the optimal strategies would only consider the average ocean currents, while for non-zero values the variance will matter. If $\alpha$ is positive the goal is to decrease the variance, i.e. to avoid worst-case scenarios in migrations. At first, it seems reasonable to assume that organisms may 312 behave as to minimize the likelihood to encounter these high cost events during migration (showing risk313 averse behaviour). However, we cannot exclude that some migrants could optimize for a more general 314 trade-off, where an increased risk of high cost would possibly lead to an increased fitness (early arrival may mean the possibility to claim, for example, the best feeding or nesting grounds). In our simulations, we found that when adopting a risk-seeking attitude, some organisms will complete their migration in a more efficient way than risk-neutral or risk-averse individuals, thus reducing their energy expenditure. In some cases, risk-seeking migrants would of course have a worse outcome than organisms more prone to risk mitigation but this behaviour may still prove advantageous in the long run.

In more general settings, behavioural experiments highlighted the potential benefits of a bold (or riskseeking) behaviour (Sundström et al. 2004; Hulthén et al. 2017): bolder fish tend to be more dominant

322 than others, have a higher ingestion rate but also suffer a higher predation rate than shy individuals. 323 Consequently, what risk attitude parameter may emerge for migration events is then a bit unclear, and 324 would probably vary from population to population, and most likely among individuals too. Moreover, we only consider here the risk-attitude as either being risk-seeking or risk-averse. In reality, the risk-parameter 
$\alpha$ is a continuous variable, and any level of risk may emerge. The reconstruction of the absolute value of the risk parameter is quite poor in our case (see supplementary material), and refining the reconstruction

328 is needed before any conclusion on the absolute risk value of the organisms can be drawn.

331 The mathematical framework proposed is general enough as it relies on few assumptions on the 332 interactions between turbulent flow and animal behaviour. The model is applied on oceanic migrations of 333 turtles but it could similarly be of use in analysing long-distance migrations of other groups such as birds, 334 whales, fish, etc. A major assumption is on the cost function (quadratic form for energy consumption and 335 linear relation with time) enabling to reduce the mathematical system to a relatively simple linear PDE 336 that is then solved with inputs on behavioural traits, current field and migration goal. A more general 337 formulation of the cost function would consider the drag force on the migrants to be quadratic - as for 338 the general case at high Reynolds number. This would then result in a metabolic cost of swimming which 339 scales as the third power of the velocity, which would then complicate the numerical solution of the model 340 as the linearized version will be no longer valid. However, for the specific case analysed here a quadratic 341 metabolic cost is a good approximation for turtles swimming (Prange 1976).

342 The cost function is also regulated by the details on the physiology of different organisms and by the way 343 organisms perform in a turbulent flow. For example, whales can easily overcome the currents and swim 344 towards their destination (Horton et al. 2011), while fish and turtles have been found to rely more on 345 ocean currents (Luschi et al. 2003; Girard et al. 2006). Consequently, migrations in turtles should give 346 greater importance to energy saving strategies when compared to whales. This is a prediction that could 347 be tested when accurate tagging data become available in the future.

348 The feeding regime of organisms may also influence energy constraints during migrations. Some species 349 can feed and replenish energy reserves while migrating (Åkesson and Hedenström 2007; Stamation et al. 350 2007). Others such as green turtles cannot feed in the open ocean, and therefore need to manage 351 cautiously their energy expenditure (Carr and Goodman 1970). The amount of control velocity an 352 individual need to invest during migrations could be also regulated by the extent of the geographic shift. 353 For example, in migrations towards high latitudes, seasonality is important and therefore a precise timing 354 may be necessary to ensure sufficient time to complete tasks such as reproduction or feeding before it is 355 time to migrate again (Both et al. 2010). When the migration is in more temperate regions, the timing may 
be less critical, and the constraints on arrival time less important. Thanks to the recent effort in assembling

357 global database on movement patterns (e.g. Sequeira et al. (2018), these hypotheses could now be tested 358 with the model by inferring behavioural traits across different biogeographical regions.

359 Organisms can in reality swim or fly in a three dimensional space and not only in a horizontal plane. In the 360 case of sea turtles, this approximation is valid as they remain the vast majority of the migration close to 361 the surface (Hays et al. 2001), where the vertical structure of horizontal currents does not change much. 362 However, for other species diving deeper or flying, individuals may select the area with winds or currents 363 that minimize migration cost (Gauthreaux 1991), subsequently optimizing their migration route in a 3D 364 space rather than a 2D horizontal plane.

\section{Statistical inference}

367 The method used for the statistical inference of the behavioural traits performs reasonably well in case 368 of strong flows, while reconstruction errors increase when conditions in ocean currents are weaker. This 369 is not unexpected as weaker ocean currents yield weaker constraints in the migration model hence lower 370 accuracy of the reconstruction algorithm. Different functions have been tested in our analyses of the 371 river case and the results shown here are for the method that provides the best results. However, aiming 372 at reconstruction of behavioural traits from tracking observations, further analyses are required to 373 systematically assess the performance under more general environmental conditions. The algorithm 374 used here (Nelder-Mead simplex algorithm, (Lagarias et al. 1998)) is robust and relatively time-efficient, 375 but other algorithms may be more efficient in the case of local minima leading to inaccurate behavioural 376 traits reconstructions. Finally, the performance of the log-likelihood, as all parameter estimation 377 methods, depends on the type of observations available. Hence, quality observations with many GPS378 data points are more likely to yield reliable estimates (and thus, to provide us with insights on the 379 behavioural ecology of long-distance migrants) than observations with lower resolution.

\section{Acknowledgements}

381 This work was supported by the Centre for Ocean Life, a VKR Centre of excellence funded by the Villum 382 Foundation. We thank Susanne Åkesson and Paolo Luschi for the discussions on turtle migrations that 383 motivated the approach presented in the manuscript. 


\section{References}

Aarestrup K, Okland F, Hansen MM, et al (2009) Oceanic Spawning Migration of the European Eel (Anguilla anguilla). Science (80- ) 325:1660-1660. https://doi.org/10.1126/science.1178120

Akesson S, Broderick AC, Glen F, et al (2003) Navigation by green turtles: which strategy do displaced adults use to find Ascension Island? Oikos 103:363-372. https://doi.org/10.1034/j.16000706.2003.12207.x

Åkesson S, Hedenström A (2007) How Migrants Get There: Migratory Performance and Orientation. Bioscience 57:123-133. https://doi.org/10.1641/B570207

Alerstam T (2001) Detours in Bird Migration. J Theor Biol 209:319-331. https://doi.org/10.1006/jtbi.2001.2266

Alerstam T (2011) Optimal bird migration revisited. J Ornithol 152:5-23. https://doi.org/10.1007/s10336011-0694-1

Alerstam T, Hedenstrom A, Åkesson S (2003) Long-distance migration: evolution and determinants. Oikos 103:247-260. https://doi.org/10.1034/j.1600-0706.2003.12559.x

Alerstam T, Lindström Å (1990) Optimal Bird Migration: The Relative Importance of Time, Energy, and Safety. In: Bird Migration. Springer Berlin Heidelberg, pp 331-351

Bellman R (1954) The Theory of Dynamic Programming. Bull Am Math Soc 60:503-515. https://doi.org/10.1090/S0002-9904-1954-09848-8

Bischof R, Loe LE, Meisingset EL, et al (2012) A migratory northern ungulate in the pursuit of spring: Jumping or surfing the green wave? Am Nat 180:407-424. https://doi.org/10.1086/667590

Bloch R, Bruderer B (1982) The air speed of migrating birds and its relationship to the wind. Behav Ecol Sociobiol 11:19-24. https://doi.org/10.1007/BF00297661

Block BA, Jonsen ID, Jorgensen SJ, et al (2011) Tracking apex marine predator movements in a dynamic ocean. Nature 475:86-90. https://doi.org/10.1038/nature10082

Bonfil R, Meÿer M, Scholl MC, et al (2005) Transoceanic Migration, Spatial Dynamics, and Population Linkages of White Sharks. Science (80- ) 310:100-103. https://doi.org/10.1126/science.1114898

Both C, Van Turnhout CAM, Bijlsma RG, et al (2010) Avian population consequences of climate change are most severe for long-distance migrants in seasonal habitats. Proc R Soc B Biol Sci 277:12591266. https://doi.org/10.1098/rspb.2009.1525

Bryson AE, Ho Y-C (1975) Applied optimal control

Byron CJ, Burke BJ (2014) Salmon ocean migration models suggest a variety of population-specific strategies. Rev Fish Biol Fish 24:737-756. https://doi.org/10.1007/s11160-014-9343-0

Cardona L, Martins S, Uterga R, Marco A (2017) Individual specialization and behavioral plasticity in a long-lived marine predator. J Exp Mar Bio Ecol 497:127-133.

https://doi.org/10.1016/j.jembe.2017.09.021 
Carmel Y, Ben-Haim Y (2005) Info-gap robust-satisficing model of foraging behavior: Do foragers optimize or satisfice? Am Nat 166:633-641. https://doi.org/10.1086/491691

Carr A (1987) New Perspectives on the Pelagic Stage of Sea Turtle Development New Perspectives on the Pelagic Stage of Sea Turtle Development. Conserv Biol 1:103-121

Carr A, Goodman D (1970) Ecologic Implications of Size and Growth in Chelonia. Copeia 783. https://doi.org/10.2307/1442334

Cerritelli G, Bianco G, Santini G, et al (2019) Assessing reliance on vector navigation in the long-distance oceanic migrations of green sea turtles. Behav Ecol 30:68-79. https://doi.org/10.1093/beheco/ary166

Clapham PJ, Mayo CA (1987) Reproduction and recruitment of individually identified humpback whales, Megaptera novaeangliae, observed in Massachusetts Bay, 1979-1985. Can J Zool 65:2853-2863. https://doi.org/10.1139/287-434

Cole ST, Wortham C, Kunze E, Owens WB (2015) Eddy stirring and horizontal diffusivity from Argo float observations: Geographic and depth variability. 1-9. https://doi.org/10.1002/2015GL063827.Received

Comincioli V (2010) Biomatematica: interazioni tra le scienze della vita e la matematica

Dalleau M, Kramer-Schadt S, Gangat Y, et al (2019) Modeling the emergence of migratory corridors and foraging hot spots of the green sea turtle. Ecol Evol ece3.5552. https://doi.org/10.1002/ece3.5552

De Luca G, Mariani P, MacKenzie BR, Marsili M (2014) Fishing out collective memory of migratory schools. J R Soc Interface 11:. https://doi.org/10.1098/rsif.2014.0043

Dingle H (2014) Migration: The Biology of Life on the Move. Second Edition.

Dvijotham K, Todorov E (2011) A Unifying Framework for Linearly Solvable Control. In: Proceedings of the Twenty-Seventh Conference Annual Conference on Uncertainty in Artificial Intelligence (UAI-11). AUAI Press, Corvallis, Oregon, pp 179-186

Fagan WF, Lewis MA, Auger-Méthé M, et al (2013) Spatial memory and animal movement. Ecol Lett 16:1316-1329. https://doi.org/10.1111/ele.12165

Feder ME, Garland T, Marden JH, Zera AJ (2010) Locomotion in Response to Shifting Climate Zones: Not So Fast. Annu Rev Physiol 72:167-190. https://doi.org/10.1146/annurev-physiol-021909-135804

Gauthreaux SAJ (1991) The Flight Behavior of Migrating Birds in Changing Wind Fields: Radar and Visual Analyses. Am Zool 31:187-204. https://doi.org/10.1093/icb/31.1.187

Girard C, Sudre J, Benhamou S, et al (2006) Homing in green turtles Chelonia mydas: oceanic currents act as a constraint rather than as an information source. Mar Ecol Prog Ser 322:281-289. https://doi.org/10.3354/meps322281

Hays GC (2017) Ocean currents and marine life. Curr Biol 27:R470-R473. https://doi.org/10.1016/j.cub.2017.01.044

Hays GC, Åkesson S, Broderick AC, et al (2001) The diving behaviour of green turtles undertaking oceanic migration to and from Ascension Island: dive durations, dive profiles and depth distribution. J Exp 
Biol 204:4093-4098

Hays GC, Åkesson S, Broderick AC, et al (2003) Island-finding ability of marine turtles. Proc R Soc London Ser B Biol Sci 270:S5-S7. https://doi.org/10.1098/rsbl.2003.0022

Hays GC, Christensen A, Fossette S, et al (2014a) Route optimisation and solving Zermelo's navigation problem during long distance migration in cross flows. Ecol Lett 17:137-143. https://doi.org/10.1111/ele.12219

Hays GC, Mortimer JA, lerodiaconou D, Esteban N (2014b) Use of Long-Distance Migration Patterns of an Endangered Species to Inform Conservation Planning for the World's Largest Marine Protected Area. Conserv Biol 28:1636-1644. https://doi.org/10.1111/cobi.12325

Hedenström A (2003) Optimal migration strategies in animals that run: A range equation and its consequences. Anim Behav 66:631-636. https://doi.org/10.1006/anbe.2003.2234

Horton TW, Holdaway RN, Zerbini AN, et al (2011) Straight as an arrow: humpback whales swim constant course tracks during long-distance migration. Biol Lett 674-679. https://doi.org/10.1098/rsbl.2011.0279

Howard RA, Matheson JE (1972) Risk-Sensitive Markov Decision Processes. Manage Sci 18:356-369. https://doi.org/10.1287/mnsc.18.7.356

Hulthén K, Chapman BB, Nilsson PA, et al (2017) A predation cost to bold fish in the wild. Sci Rep 7:3-7. https://doi.org/10.1038/s41598-017-01270-w

Jørgensen C, Ernande B, Fiksen $\varnothing$, Dieckmann U (2006) The logic of skipped spawning in fish. Can J Fish Aquat Sci 63:200-211. https://doi.org/10.1139/f05-210

Lagarias JC, Reeds JA, Wright MH, Wright PE (1998) Convergence Properties of the Nelder--Mead Simplex Method in Low Dimensions. SIAM J Optim 9:112-147. https://doi.org/10.1137/S1052623496303470

Lennox RJ, Chapman JM, Souliere CM, et al (2016) Conservation physiology of animal migration. Conserv Physiol 4:1-15. https://doi.org/10.1093/conphys/cov072

Luschi P, Hays GC, Del Seppia C, et al (1998) The navigational feats of green sea turtles migrating from Ascension Island investigated by satellite telemetry. Proc Biol Sci 265:2279-2284. https://doi.org/10.1098/rspb.1998.0571

Luschi P, Hays GC, Papi F (2003) A review of long-distance movements by marine turtles, and the possible role of ocean currents. Oikos 103:293-302. https://doi.org/10.1034/j.16000706.2003.12123.x

McLaren JD, Shamoun-Baranes J, Dokter AM, et al (2014) Optimal orientation in flows: providing a benchmark for animal movement strategies. J R Soc Interface 11:20140588. https://doi.org/10.1098/rsif.2014.0588

Metcalfe JD, Hunter E, Buckley AA (2006) The migratory behaviour of North Sea plaice: Currents, clocks and clues. Mar Freshw Behav Physiol 39:25-36. https://doi.org/10.1080/10236240600563404

Mueller T, O'Hara RB, Converse SJ, et al (2013) Social Learning of Migratory Performance. Science (80- ) 
Nichols WJ, Resendiz A, Seminoff J a., Resendiz B (2000) Transpacific migration of a loggerhead turtle monitored by satellite telemetry. Bulletin of Marine Science 67:937-947

$\varnothing$ ksendal B (2003) Stochastic Differential Equations. Springer Berlin Heidelberg, Berlin, Heidelberg

Prange HD (1976) Energetics of swimming of a sea turtle. J Exp Biol 64:1-12

Schott FA, McCreary JP (2001) The monsoon circulation of the Indian Ocean. Prog Oceanogr 51:1-123. https://doi.org/10.1016/S0079-6611(01)00083-0

Scott R, Marsh R, Hays GC (2014) Ontogeny of long distance migration. Ecology 95:2840-2850. https://doi.org/10.1890/13-2164.1

Sequeira AMM, Rodríguez JP, Eguíluz VM, et al (2018) Convergence of marine megafauna movement patterns in coastal and open oceans. Proc Natl Acad Sci 115:3072-3077. https://doi.org/10.1073/pnas.1716137115

Shaffer SA, Tremblay Y, Weimerskirch $\mathrm{H}$, et al (2006) Migratory shearwaters integrate oceanic resources across the Pacific Ocean in an endless summer. Proc Natl Acad Sci U S A 103:12799-12802. https://doi.org/10.1073/pnas.0603715103

Shamoun-Baranes J, Leyrer J, van Loon E, et al (2010) Stochastic atmospheric assistance and the use of emergency staging sites by migrants. Proc R Soc B Biol Sci 277:1505-1511. https://doi.org/10.1098/rspb.2009.2112

Stamation KA, Croft DB, Shaughnessy PD, Waples KA (2007) Observations of Humpback Whales (Megaptera novaeangliae) Feeding During Their Southward Migration Along the Coast of Southeastern New South Wales, Australia: Identification of a Possible Supplemental Feeding Ground. Aquat Mamm 33:165-174. https://doi.org/10.1578/AM.33.2.2007.165

Sundström LF, Petersson E, Höjesjö J, et al (2004) Hatchery selection promotes boldness in newly hatched brown trout (Salmo trutta): implications for dominance. Behav Ecol 15:192-198. https://doi.org/10.1093/beheco/arg089

Thomson KA, Ingraham WJ, Healey MC, et al (1992) The influence of ocean currents on latitude of landfall and migration speed of sockeye salmon returning to the Fraser River. Fish Oceanogr 1:163179. https://doi.org/10.1111/j.1365-2419.1992.tb00035.x

Weinrich M (1998) Early Experience in Habitat Choice by Humpback Whales (Megaptera novaeangliae). J Mammal 79:163-170. https://doi.org/10.2307/1382851

Whittle P (1990) Risk-sensitive optimal control. Wiley

Wolf M, van Doorn GS, Leimar O, Weissing FJ (2007) Life-history trade-offs favour the evolution of animal personalities. Nature 447:581-584. https://doi.org/10.1038/nature05835

Zermelo E (1931) Über das Navigationsproblem bei ruhender oder veranderlicher Windverteilung. 
Figure 2 Tracks of migrants crossing a $1 \mathrm{~km}$-long shear flow. The time between two data points is 1 minute, and the colour code shows the ratio between advective and control speed $v / w$. a) Tracks for different values of $\alpha: 1,0.1,0,-0.1,1,10$ (from left to right). b) Tracks for different values of $\beta / \gamma: 10^{-2}, 10^{-1}, 1,10$, 100 (from left to right). c) Representation of the shear flow used.

Figure 2 Tracks generated by the model in the Atlantic with random behavioural parameters. The arrival area is the red ellipse, and the departure points is the red circle (A, Ascension Island). Background is the current speed (in $\mathrm{m} / \mathrm{s}$ ), and the track colours represent the control velocity (in $\mathrm{m} / \mathrm{s})$. Dashed black line is an original migration track of green turtle, reproduced from Cerritelli et al. (2019).

Figure 3 Tracks generated by the model for organisms migrating between Diego García (DG, red circle C) and the Somalian coast (red dashed ellipse) with random behavioural parameters. Background is the current speed (in $\mathrm{m} / \mathrm{s}$ ), and the track colours represent the control velocity (in $\mathrm{m} / \mathrm{s})$. Dashed black lines are original migration tracks of green turtles, reproduced from Hays et al. (2014b).

Figure 4 Top: Reconstruction of the sign of the risk parameter $\alpha$ and of the adimensionalized cost ratio in the theoretical cases. For the risk attitude: the dark share of the chart represents the misreconstructed signs, the dark grey the correct positive (risk-averse) reconstruction, and the light grey the correct negative (risk-seeking) reconstructions. Bottom: For the cost ratio: the histograms were constrained between -1 and 1, and all more extreme values were assigned to -1 (if negative) or 1 (if positive). Each bar represents $10 \%$ of error.

Figure 5 Top: Reconstruction of the sign of the risk parameter $\alpha$ and of the adimensionalized cost ratio in the oceanic cases. For the risk attitude: the dark share of the chart represents the misreconstructed signs, the dark grey the correct positive (risk-averse) reconstruction, and the light grey the correct negative (risk-seeking) reconstructions. Bottom: For the cost ratio: the histograms were constrained between -2 and 2, and all more extreme values were assigned to -2 (if negative) or 2 (if positive). Each bar represents $10 \%$ of error. 